Arq. Bras. Med. Vet. Zootec., v.62, n.1, p.23-29, 2010

\title{
Influência do treinamento aeróbio sobre o cortisol e glicose plasmáticos em equinos
}

\author{
[Influence of the aerobic training on cortisol and glucose levels in horses] \\ G.C. Ferraz, A.R. Teixeira-Neto, M.C. Pereira, R.L. Linardi, \\ J.C. Lacerda-Neto, A. Queiroz-Neto \\ Faculdade de Ciências Agrárias e Veterinárias - UNESP \\ Via de acesso Prof. Paulo Donato Castellane s/n \\ 14884-900 - Jaboticabal, SP
}

\begin{abstract}
RESUMO
Estudou-se a resposta do cortisol e da glicemia em 12 equinos da raça Puro Sangue Árabe destreinados $\left(\mathrm{T}_{0}\right)$ por oito meses e submetidos a um período de 90 dias de treinamento aeróbio $\left(\mathrm{T}_{90}\right)$. Para avaliação dos efeitos do treinamento, empregou-se teste ergométrico constituído de exercício progressivo em esteira rolante, acompanhado por colheitas de sangue 15 segundos antes do término de cada etapa de esforço. A velocidade (intensidade) do treino foi definida como sendo $80 \%$ da $\mathrm{V}_{4}$ (velocidade na qual a lactacidemia atinge $4 \mathrm{mmol} / \mathrm{L}$ ). Adicionalmente, no último mês de treinamento, foi instituído, uma vez por semana, exercício com velocidades variáveis, chamado "fartlek". Após 90 dias de treinamento, a concentração plasmática de cortisol elevou-se e após o teste de esforço (20min), houve aumento da glicemia. Este resultado reflete a possibilidade de adaptação ao treinamento. Conclui-se que o cortisol plasmático pode ser utilizado como ferramenta na avaliação de um programa de treinamento em equinos.
\end{abstract}

Palavras-chave: eqüino, programa de treinamento, cortisol, glicose

\begin{abstract}
Cortisol and glucose responses were evaluated in 12 Arabian (PSA) horses submitted to a detraining period of eight months $\left(T_{0}\right)$ and to 90 days of aerobic training $\left(T_{90}\right)$. For the evaluation of the effect of training, a standardized incremental exercise test in a treadmill was used. Fifteen seconds before the ending of each effort step, blood samples were collected. The speed (intensity) of the training was defined as being $80 \%$ of the $V_{4}$ (speed at which the blood lactate concentration reaches $4 \mathrm{mmol} / \mathrm{L}$ ). Additionally, in the last month of training, velocity play, a type of exercise with varying velocities called "fartlek" was instituted, once a week. Results showed that after 90 days of training, the plasmatic concentrations of cortisol and glucose increased when compared to the untrained horses. This result reflects the possibility of adaptation to the training. The blood cortisol levels may be used as a tool for the evaluation of a training program in horses.
\end{abstract}

Keywords: equine, traing program, cortisol, glucose

\section{INTRODUÇÃO}

A melhoria do desempenho atlético por meio da utilização de programas racionais de treinamento eleva a capacidade de realização de trabalho físico, pois provoca adaptações aos aumentos na sobrecarga de esforço que são impostos durante a temporada de eventos esportivos, diminuindo a possibilidade de injúrias especialmente do sistema músculo-esquelético. Assim, a busca de soluções tecnológicas que tenham como princípio a utilização sustentável dos animais deve ser tentada em pesquisas científicas da fisiologia do exercício equino.

No cotidiano da clínica médica esportiva, são frequentes os períodos de repouso destinados à recuperação de cavalos enfermos, o que, invariavelmente, leva à perda do condicionamento físico. Dessa maneira, quando

Recebido em 8 de setembro de 2009

Aceito em 2 de fevereiro de 2010

E-mail: guilherme.de.ferraz@terra.com.br 
ocorre o retorno desses atletas às pistas, o negligenciamento da determinação de seu estado atlético pode acarretar na redução da vida útil do atleta, causando perdas financeiras (Ferraz et al., 2007).

O exercício físico representa o estímulo estressante mais fisiológico que existe, pois submete o organismo a desafios temporários na sua homeostasia (Cayado et al., 2006). Quando o esforço físico torna-se sistemático e contínuo, com aumento gradual da intensidade, intercalado a períodos de repouso, é denominado treinamento, sendo que o maior objetivo deste processo é provocar adaptações fisiológicas que aprimorem o desempenho atlético (GraafRoelfsema et al., 2007).

O termo estresse descreve o estado de um organismo sob influência de fatores externos e internos, que ameaçam ou alteram a homeostase, e as mudanças adaptativas ocorridas em decorrência desses estímulos são cognitivas, comportamentais e corporais. Uma vez que certo limiar de esforço físico for ultrapassado, ocorrerá efeito sistêmico com envolvimento do sistema nervoso periférico e central, e especialmente o sistema nervoso autônomo simpático e o eixo hipotálamo-hipófise-adrenal (EHHA) são ativados (Graaf-Roelfsema et al., 2007).

A sensibilização do eixo EHHA libera cortisol, principal glicocorticoide produzido pelos equinos, que controla largo espectro de funções metabólicas. Dentre elas, destaca-se o metabolismo energético por meio da gliconeogênese, sendo que este hormônio inibe a utilização de glicose pelos tecidos, priorizando a mobilização para o sistema nervoso central (SNC) e permitindo que, durante o exercício, a glicemia permaneça constante. Segundo Wilmore e Costill (1994), esse efeito previne o início da fadiga central.

Os resultados de alguns estudos (Lindner et al., 2002; Graaf-Roelfsema et al., 2007) indicaram que o treinamento pode afetar a concentração plasmática de cortisol antes, durante e após o exercício. Dessa maneira, essa variável fisiológica poderia contribuir como indicador confiável para avaliação da aptidão física (Ferlazzo et al., 2006). A maioria das investigações científicas, que utiliza a espécie equina como modelo experimental, compara animais bem treinados e experientes com aqueles em início de treinamento específico para determinada modalidade equestre. Cayado et al. (2006), ao estudarem cavalos de hipismo, demonstraram haver uma resposta estressante padrão, sendo que diferentes estágios de treinamento induziam efeitos distintos sobre o EHHA.

De uma maneira geral, poucos estudos verificaram a resposta do EHHA em equinos destreinados que retornaram ao treinamento (Graaf-Roelfsema et al., 2007). O propósito deste estudo foi avaliar as alterações no cortisol e sua possível utilização como indicador para avaliação de equinos submetidos a treinamento.

\section{MATERIAL E MÉTODOS}

Utilizaram-se 12 equinos, machos (cinco) e fêmeas (sete), com idade média de 8,6 $\pm 3,3$ anos, Puro Sangue Árabe (PSA), todos provenientes do setor de equinocultura da Faculdade de Ciências Agrárias e Veterinárias - Unesp, Jaboticabal, com média de peso de $391 \pm 25,4 \mathrm{~kg}$. Os animais foram submetidos a exame clínico completo e, estando aparentemente hígidos, foram selecionados para comporem os grupos experimentais. Os equinos permaneceram durante oito meses sem atividade física, o que correspondeu ao período de destreinamento. Diariamente era fornecido feno de coast-cross (Cynodon sp.), sal mineralizado (Agromix) água e pastagem natural (Brachiaria sp.) ad libitum.

Para determinação das quantidades diárias de ração comercial (Nutriage Mix Guabi) fornecida (2,5 a 3,8kg/animal), os animais foram avaliados mensalmente por meio do protocolo proposto por Henneke et al. (1983). Embora metade dos animais tenha recebido suplementação com $75 \mathrm{~g}$ de creatina mono-hidratada (Vetnil), diariamente, durante o período de treinamento, o efeito deste tratamento não se mostrou estatisticamente significativo sobre as variáveis estudadas. Dessa forma, na composição dos grupos experimentais, consideraram-se apenas dois grupos de animais, destreinados $\left(\mathrm{T}_{0}\right)$ e treinados por 90 dias $\left(\mathrm{T}_{90}\right)$.

Estudaram-se os efeitos do treinamento sobre o cortisol plasmático e a glicemia de equinos. Para tanto, compararam-se essas variáveis em cavalos 
após um período de inatividade de 8 meses e depois de três meses de treinamento.

O programa de treinamento foi realizado numa sala climatizada, exclusivamente em esteira rolante (Galloper 5500 - Sahinco). Este protocolo foi adotado devido às afirmações de Evans (2000), que relata efetividade nos treinamentos realizados em esteira. Previamente ao início do programa de treinamento, os equinos foram submetidos a um período de 30 dias de adaptação ao manejo. A velocidade (intensidade) do treino foi definida como sendo $80 \%$ da $\mathrm{V}_{4}$ (velocidade que a lactacidemia atinge $4 \mathrm{mmol} / \mathrm{L}$ ). Para a determinação da $\mathrm{V}_{4}$, os cavalos eram submetidos a um teste incremental. Dessa forma, determinou-se, por meio de análise de regressão exponencial, a velocidade na qual a lactacidemia alcançava o limiar de lactato $(4 \mathrm{mmol} / \mathrm{L})$. Ao final de cada período de treinamento (30 dias), realizava-se um novo teste obtenção de uma nova $\mathrm{V}_{4}$. Assim, mensalmente determinava-se a velocidade de treinamento para cada animal.

A frequência do treinamento compreendeu a realização de exercícios três vezes por semana (dias alternados). No primeiro mês de treinamento, cada animal percorreu, em média, $10 \mathrm{~km}$ em $42 \mathrm{~min}$. No segundo mês, a distância percorrida em cada exercício aumentou para $15 \mathrm{~km}$, com duração média de $56 \mathrm{~min}$. No último mês, todos os cavalos percorreram $20 \mathrm{~km}$ por seção, com duração média de 55 min. Adicionalmente, nesse mês foi instituído, uma vez por semana, treinamento do tipo jogo de velocidade, ou seja, "fartlek". Nesse exercício, foi empregado um protocolo que se iniciava com um período de aquecimento a $4,0 \mathrm{~m} / \mathrm{s}$ durante $10 \mathrm{~min}$. Na segunda etapa, a esteira era inclinada para $5 \%$ e a velocidade aumentada para $8 \mathrm{~m} / \mathrm{s}$, com duração de $10 \mathrm{~min}$. Na etapa seguinte, a velocidade da esteira e a duração do exercício eram reduzidas para $2,5 \mathrm{~m} / \mathrm{s}$ e $5 \mathrm{~min}$, respectivamente, sem inclinação. A seguir, a esteira era inclinada a $5 \%$, e a velocidade aumentada para $10 \mathrm{~m} / \mathrm{s}$ por $5 \mathrm{~min}$. Novamente, reduzia-se a velocidade para $2,5 \mathrm{~m} / \mathrm{s}$ por $5 \mathrm{~min}$, sem inclinação. A velocidade da esteira era novamente aumentada para $5,0 \mathrm{~m} / \mathrm{s}$, com inclinação de $10 \%$ e duração de 20min. Logo em seguida, aumentava-se a velocidade para $12 \mathrm{~m} / \mathrm{s}$, sem inclinação, com duração de $3 \mathrm{~min}$. Os animais finalizavam a atividade física com um período de desaquecimento a $2,0 \mathrm{~m} / \mathrm{s}$ por $10 \mathrm{~min}$, sem inclinação.

Os equinos foram adaptados ao exercício em esteira rolante de alto desempenho e submetidos ao exercício teste (ET) com duração de 30min. Para tanto, empregou-se exercício de aquecimento durante $4 \mathrm{~min}$ a velocidade de $4,0 \mathrm{~m} / \mathrm{s}$, a qual foi incrementada a intervalos de $2 \mathrm{~min}$, para 6,$0 ; 8,0$ e $10 \mathrm{~m} / \mathrm{s}$. A partir dessa etapa de esforço máximo, procedeu-se à desaceleração, retomando a velocidade para $3,0 \mathrm{~m} / \mathrm{s}$, por $20 \mathrm{~min}$, que correspondeu ao período de desaquecimento ativo. Toda a fase de esforço físico, com incremento da velocidade, foi realizada com a esteira a $10 \%$ de inclinação.

Para obtenção das amostras de sangue, foi criado um procedimento operacional padrão (POP) que estabeleceu procedimentos adequados para a colheita, processamento e armazenamento. As colheitas de sangue foram realizadas 15 segundos do término de cada etapa de velocidade do exercício teste. Previamente à realização do exercício, os animais foram tricotomizados e assepticamente preparados para venocateterização, utilizando-se a veia jugular esquerda como ponto de colheita. Acoplou-se ao cateter intravenoso um tubo extensor para facilitar as colheitas com o animal em movimento. Após cada colheita, todo o conjunto era lavado com solução de heparina a 2,5\%. Pelo procedimento, desprezavam-se $2,0 \mathrm{~mL}$ de sangue advindos do início de cada colheita.

Para determinação do cortisol e da glicemia, utilizaram-se amostras colhidas na fase de exercício. $\mathrm{Na}$ fase de desaquecimento ativo, quantificaram-se as variáveis nos momentos 20 e $30 \mathrm{~min}$.

Dez mililitros de sangue foram obtidos em tubos Vacutainer contendo heparina sódica e imediatamente centrifugados sob refrigeração e o plasma congelado a $-20^{\circ} \mathrm{C}$. Para dosagem do cortisol, empregou-se kit comercial de radioimunoensaio em fase sólida (Coat-a-count Diagnostic Prod. Corp.). As amostras foram analisadas em duplicatas, e os coeficientes de variação intra e interensaio foram de $6,31 \pm 1,5 \%$ e $9,2 \pm 1,4 \%$.

As amostras de sangue $(3,0 \mathrm{~mL})$ destinadas à dosagem de glicose foram processadas com 
anticoagulante contendo inibidor da glicólise, fluoreto de potássio, centrifugadas e imediatamente analisadas por espectofotometria por meio de kit comercial baseado na reação hexocinase (HK - Sigma Chemical). As amostras foram analisadas em triplicatas, e os coeficientes de variação intra e interensaio foram de $5,1 \pm 1,3 \%$ e $7,3 \pm 1,8 \%$, respectivamente.

A análise estatística foi realizada utilizando-se o programa computacional SAS System for Windows v8, e os resultados foram apresentados como média \pm erro-padrão da média. Para a comparação do cortisol e da glicemia obtidas no exercício teste, com o objetivo de avaliar as diferenças dentro de cada grupo experimental, foi realizada análise de variância. Para as diferenças estatísticas detectadas no teste F, realizou-se a comparação dos valores médios obtidos em cada grupo, tanto na fase de exercício como no desaquecimento ativo, por meio do teste de Tukey. Para todas as análises realizadas, estabeleceu-se como nível de significância $\mathrm{P} \leq 0,05$.

\section{RESULTADOS E DISCUSSÃO}

Durante o período de 90 dias de treinamento, os cavalos realizaram atividade física abaixo do limiar de lactato $(4 \mathrm{mmol} / \mathrm{L}), 80 \% \mathrm{~V}_{4}$, fazendo com que o esforço físico fosse exclusivamente aeróbio. O principal objetivo desse tipo de atividade, também nomeada fase de resistência ou treinamento base, foi melhorar a capacidade aeróbia e a resistência do sistema músculoesquelético (D'Angelis et al., 2005; Rivero, 2007), reduzindo a frequência das enfermidades do aparelho locomotor.

Com relação ao cortisol, no repouso não houve diferença entre os grupos experimentais (Fig. 1A). A mesma resposta é observada quando se comparam atletas da espécie humana destreinados com aqueles submetidos à treinamento aeróbio. A concentração basal de cortisol pode estar igual nas duas condições, indicando perda de sensibilidade da glândula adrenal em decorrência do treinamento (Duclos et al., 2001), pois, em indivíduos treinados, geralmente verifica-se que as concentrações do hormônio adrenocorticotrófico (ACTH) estão elevadas (Heitkamp et al., 1998). Adicionalmente, a redução da sensibilidade da glândula adrenal pode indicar adaptação bem- sucedida ao programa de treinamento (GraafRoelfsema et al., 2007).

Outro aspecto que pode ser abordado é a resposta do EHHA frente ao exercício agudo, protocolo do teste utilizado neste estudo, um esforço incremental com duração de 10 minutos. Sempre comparando ao repouso, a Fig. 1A revela que a concentração plasmática de cortisol elevou-se $(\mathrm{P}<0,0001)$ conforme se submeteram os animais do grupo $\mathrm{T}_{90}$ ao esforço. A maioria dos estudos relata que o exercício agudo provoca aumento do cortisol tanto em humanos (Consitt et al., 2002) como nos equinos (McKeever, 2002; Cayado et al., 2006), sendo que essa elevação está relacionada com a intensidade e a duração da atividade física. Nos exercícios curtos (4min), porém realizados em intensidades altas, a resposta do córtex da adrenal é menor quando comparada àquelas observadas com intensidades moderadas, mas ocorridas em exercícios de duração maior (12min) (Nagata et al., 1999). Esses autores concluíram que a elevação do cortisol está mais relacionada à duração do que à velocidade (intensidade) do esforço.

Quanto à ação do exercício sobre o EHHA, observa-se que os cavalos da raça Puro Sangue Árabe submetidos ao esforço progressivo em esteira rolante tiveram resposta semelhante a outros tipos de estímulos como corrida (Nagata et al., 1999), concurso completo de equitação (Linden et al., 1991) e hipismo (Cayado et al., 2006). Comparativamente, em atletas da espécie humana, esta elevação também ocorre (Consitt et al., 2002), podendo, tanto em indivíduos da espécie humana como em equinos, atingir o dobro da concentração basal (Graaf-Roelfsema et al., 2007). A Fig. 1A revela que houve aumento do cortisol para o grupo $\mathrm{T}_{90}$, de $180 \pm 8$ para $314 \pm 27 \mathrm{nmol} / \mathrm{L}$ (aos 30min, $\mathrm{P}<0,0001)$.

No presente estudo, utilizaram-se animais destreinados, que tiveram contato com o esquema de treinamento em esteira somente durante o período experimental. Interessante foi que o treinamento por um período de 90 dias elevou $(\mathrm{P}<0,0001)$ o cortisol a partir da etapa de intensidade máxima do ET (Tab. 1A). Este achado é diferente daqueles obtidos por Cayado et al. (2006), que verificaram em equinos de hipismo menores concentrações de cortisol, indicando redução do estresse para animais melhores condicionados. Uma possível 
explicação para essa discrepância seria a de que esses autores compararam animais altamente experientes com outros de experiência intermediária, porém também treinados e já adaptados aos fatores envolvidos no processo de treinamento. Diferentemente, os resultados apresentados neste experimento sugerem que, após um período longo de inatividade, ocorre, após o reinício do treinamento, resposta do EHHA que eleva a produção de cortisol, indicando que, neste caso ainda não ocorreu adaptação ao esforço físico.
Outra explicação plausível para elevação do cortisol seria a introdução, aos 60 dias de treinamento, de exercício do tipo "fartlek". Segundo Evans (2000), a utilidade deste tipo de treino, que alterna intensidades de esforço leves com intensas, é aumentar a variável velocidade dos equinos que estão realizando treinamento aeróbio. Portanto, com o aumento do esforço (estímulo), nesta fase do treinamento, houve maior resposta do EHHA quando comparado ao grupo destreinado (Fig. 1B).
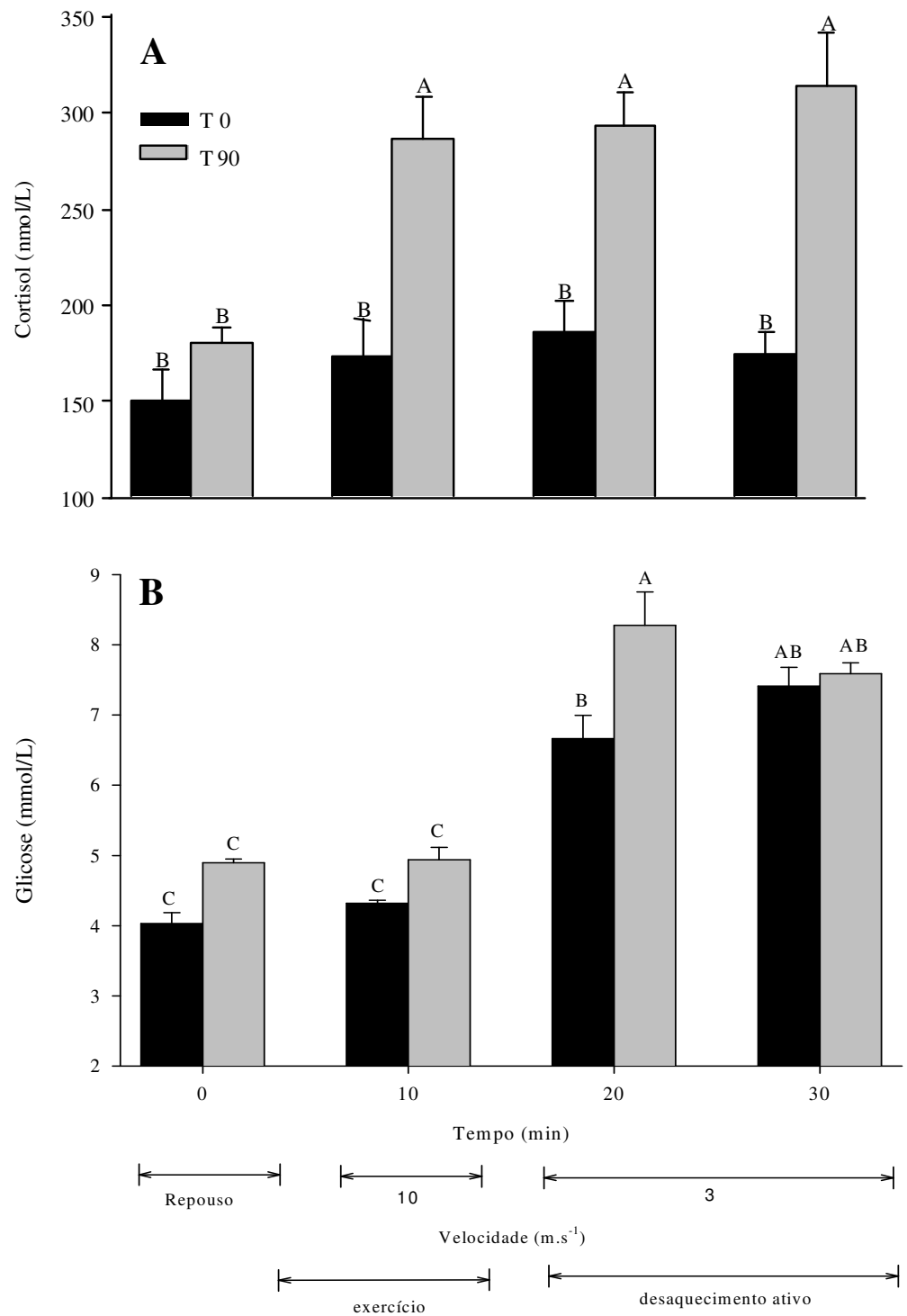

Figura 1. Concentração de cortisol plasmático (A) e glicemia (B) em equinos da raça Puro Sangue Árabe (PSA) destreinados $\left(\mathrm{T}_{0}\right)$ e submetidos a 90 dias de treinamento aeróbio $\left(\mathrm{T}_{90}\right)$. $\mathrm{N}=12$. Letras diferentes indicam alterações entre os grupos. 
McKeever e Gordon (2007) relataram que concentrações elevadas de cortisol durante o treinamento podem indicar adaptação, tendo em vista a atividade anti-inflamatória desse hormônio, sendo um efeito benéfico e preventivo à ação de substâncias pró-inflamatórias que são produzidas pelo exercício agudo.

Do ponto de vista prático, é importante enfatizar que esses achados indicam a importância de períodos de treinamento aeróbio relativamente longos para os cavalos que estão retornando de períodos prolongados de recuperação devido a diversas entidades mórbidas inerentes à atividade esportiva equestre. Mesmo parecendo óbvio, a contribuição deste trabalho é indicar que aumentos na intensidade de esforço são estímulos que provocam resposta estressante e devem ser prescritos de maneira racional, respeitando a individualidade de cada atleta. Dessa maneira, o manejo será preventivo, diminuindo o risco de lesões músculoesqueléticas, conceito de treinamento sustentável.

Outra função do cortisol é controlar o metabolismo de carboidratos, na forma de glicogênio muscular e glicose plasmática que são importantes substratos mobilizados para a contração da musculatura esquelética. Neste estudo, avaliou-se a dinâmica da glicemia frente ao exercício intenso. A concentração de glicose plasmática (Fig. 1B) elevou-se conforme foram incrementadas as etapas de esforço em todos os grupos experimentais. Elevações na glicemia durante e após o exercício intenso são frequentemente observadas, sendo um achado consistente com a literatura (Trilk et al., 2002; Simões et al., 2003). Com o exercício intenso, ocorre aumento da glicemia devido ao incremento da glicogenólise e neoglicogênese hepática provocadas pela ação tanto do cortisol (Simões et al., 2003) como da atividade adrenérgica (Geor et al., 2000). Quanto ao tipo de exercício, existem diferenças significantes quando ocorre comparação entre o esforço intenso de curta duração e o moderado (resistência). Fernandes e Larsson (2000) estudaram equinos submetidos a provas de enduro de $30 \mathrm{~km}$ e encontraram redução da glicemia ao término do esforço.

A Fig. 1B também revela, quando se avalia o efeito do treinamento, que, após o teste de esforço (20min), houve aumento da glicemia nos animais do grupo $\mathrm{T}_{90}$. Este resultado reflete a possibilidade de adaptação ao treinamento, pois existe clara tendência para a manutenção normoglicêmica na tentativa de prevenir o início da fadiga central, favorecendo o desempenho neuromuscular (Nybo, 2003).

\section{CONCLUSÕES}

O treinamento produz alterações na resposta do EHHA, sendo que as concentrações de cortisol plasmático elevaram-se em relação ao aumento da intensidade de esforço, e esta variável fisiológica pode ser utilizada criteriosamente como ferramenta que indica estímulo estressante adicional num programa de treinamento.

\section{REFERÊNCIAS BIBLIOGRÁFICAS}

CAYADO, P.; MUÑOZ-ESCASSI, B.; DOMÃ-NGUEZ, C. et al. Hormone response to training and competition in athletic horses. Equine Vet. J., v.36, suppl., p.274-278, 2006.

CONSITT, L.A.; COPELAND, J.L.; TREMBLAY, M.S. Endogenous anabolic hormone responses to endurance versus resistance exercise and training in women. Sports Med., v.32, p.1-22, 2002.

D’ANGELIS, F.H.F.; FERRAZ, G.C.; BOLELI, I.C. et al. Aerobic training, but not creatine supplementation, alters the gluteus medius muscle. J. Anim. Sci., v.83, p.579-585, 2005.

DUCLOS, M.; CORCUFF, J.B.; PEHOURCQ, F. et al. Decreased pituitary sensitivity to glucocorticoids in endurance-trained men. Eur. $J$. Endocrinol., v.144, p.363-368, 2001.

EVANS, D.L. Training and fitness in athletic horses. Sydney: Rural Industries Research \& Development Corporation, 2000. 64p.

FERLAZZO, A.; MEDICA, P.; FAZIO, E. Hormonas y ejercicio. In: BOFFI, F.M. Fisiologia del ejercicio. Buenos Aires: InterMédica, 2006. p.153-164.

FERNANDES, W.R.; LARSSON, M.H.M.A. Alterações nas concentrações séricas de glicose, sódio, potássio, ureia e creatinina, em equinos submetidos a provas de enduro de $30 \mathrm{~km}$ com velocidade controlada. Cienc. Rural, v.30, p.393398, 2000. 
FERRAZ, G.C.; ESCODRO, P.B.; QUEIROZ NETO, A. Fisiologia do exercício equino: ferramenta para o desempenho atlético de cavalos atletas. Braz. J. Equine Med., v.12, p.6-8, 2007.

GEOR, R.J.; HINCHCLIFF, K.W.; SAMS, R.A. $\beta$-Adrenergic blockade augments glucose utilization in horses during graded exercise. $J$. Appl. Physiol., v.89, p.1086-1098, 2000.

GRAAF-ROELFSEMA， E.; KEIZER， H.A.; BREDA E.V. et al. Hormonal responses to acute exercise, training and overtraining: A review with emphasis on the horse. Vet. Q., v.29, p.82101,2007

HEITKAMP, H.C.; SCHULZ, H.; RÖCKER, K. et al. Endurance training in females: Changes in $\beta$-Endorphin and ACTH. Int. J. Sports Med., v.19, p.260-264, 1998.

HENNEKE, D.R.; POTTER, G.D.; KREIDER, J.L. et al. Relationship between condition score, physical measurements and body fat percentage in mares. Equine Vet. J., v.15, p.371-372, 1983.

LINDEN, A.; ART, T.; AMORY, H. et al. Quantitative buffy coat analysis related to adrenocortical function in horses during a threeday event competition. Zentbl. Veterinaermed. Reihe A, v.38, p.376-382, 1991.

LINDNER, A.; FAZIO, E.; MEDICA, P. et al. Effect of age, time record and $\mathrm{V}_{4}$ on plasma cortisol concentration in Standardbred racehorses during exercise. Pferdeheilkunde, v.18, p.51-56, 2002 .
McKEEVER, K.H.; GORDON, M.E. Endocrine alterations in the equine athlete. In: HINCHCLIFF, K.W.; KANEPS, A.; GEOR, G. (Eds). Equine Exercise Physiology. Philadelphia: Elsevier, 2007. p.278-304.

McKEEVER, K.H. The endocrine system and the challenge of exercise. Vet. Clin. N. Am.: Equine Pract., v.18, p.321-353, 2002.

NAGATA, S.; TAKEDA, F.; KUROSAWA, M. et al. Plasma adrenocorticotropin, cortisol and catecholamines response to various exercises. Equine Vet. J., v.30, suppl., p.570-574, 1999.

NYBO, L. CNS fatigue and prolonged exercise: effect of glucose supplementation. Med. Sci. Sports Exerc., v.35, p.589-594, 2003.

RIVERO, J.L.L. A scientific background for skeletal muscle conditioning in equine practice. J. Vet. Med. A, v.54, p.321-332, 2007.

SIMÕES, H.G.; CAMPBELL, C.S.; KUSHNICK, M.R. et al. Blood glucose threshold and the metabolic responses to incremental exercise tests with and without prior lactic acidosis induction. Eur. J. Appl. Physiol., v.89, p.603-611, 2003.

TRILK, J.L.; LINDNER, A.J.; GREENE, H.M. et al. A lactate-guided conditioning programme to improve endurance performance. Equine Vet. J., v.34, suppl., p.122-125, 2002.

WILMORE, J.H; COSTILL, D.L. Hormonal regulation of exercise. In: Physiology of sport and exercise. Champaign, IL: Human Kinetics, 1994. p.122-143. 